\title{
NRP-1 expression is strongly associated with the progression of pituitary adenomas
}

\author{
SANG WON LEE ${ }^{1 *}$, JUNG EUN LEE $^{1 *}$, CHANG YOUNG YOO ${ }^{2}$, MYOUNG SEOK KO $^{4}$, \\ CHAN SOON PARK ${ }^{3}$ and SEUNG-HO YANG ${ }^{1}$ \\ Departments of ${ }^{1}$ Neurosurgery, ${ }^{2}$ Pathology and ${ }^{3}$ Otolaryngology, St. Vincent's Hospital, \\ The Catholic University of Korea, Paldal-gu, Suwon 442-723; ${ }^{4}$ Asan Institute for \\ Life Sciences, Asan Medical Center, Seoul 138-736, Republic of Korea
}

Received April 25, 2014; Accepted July 24, 2014

DOI: $10.3892 /$ or.2014.3392

\begin{abstract}
The purpose of the present study was to identify a predictive marker associated with tumor progression or recurrence. We investigated the expression of p53, Ki-67, Bax, Bcl-2, vascular endothelial growth factor (VEGF)-A, VEGFR-1, VEGFR-2 and neuropilin-1 (NRP-1) in pituitary adenomas (PAs) with/without tumor progression during follow-up periods. We compared the expression of these molecules in primary and recurrent specimens to identify a predictive marker associated with tumor progression. Nineteen patients had no progression for more than 5-years of follow-up. Nine patients had tumor progression within 5 years of their first transsphenoidal surgery (TSS) surgery and underwent re-TSS for treating progression of adenoma. Tumor size was larger and involvement of the cavernous sinus was more frequent in the progression group than these variables in the no progression group. A strong association was observed between NRP-1 expression and tumor progression. No significant risk for developing tumor progression was associated with Ki-67, p53, Bax, Bcl-2, VEGFR-1, VEGFR-2, or VEGF-A expression. Four of nine patients showed strong NRP-1 immunoreactivity in progression specimens. Negative NRP-1 immunoreactivity in the initial specimens was converted into strong positivity in the progression specimens of five patients. NRP-1 could be a relevant PA marker of progression and could be a potential target for medical therapy.
\end{abstract}

Correspondence to: Dr Seung-Ho Yang, Department of Neurosurgery, St. Vincent's Hospital, College of Medicine, The Catholic University of Korea, 93-6 Chi-dong, Paldal-gu, Suwon 442-723, Republic of Korea

E-mail: 72ysh@catholic.ac.kr

*Contributed equally

Key words: pituitary adenoma, disease progression, neuropilin-1, immunohistochemistry, angiogenesis

\section{Introduction}

Pituitary adenomas (PAs) are the most common tumors of the pituitary, frequently asymptomatic and discovered at autopsy in up to $15 \%$ of patients. PAs comprise $~ 7 \%$ of intracranial tumors (1). Patients present with signs of mass effect (visual loss), hormone overproduction, or hypopituitarism. These benign tumors have a low growth rate but some recur after surgery. Tumor size, invasion and the adequacy of resection are important risk factors for recurrence or progression (2).

Previously investigated proliferative markers include $\mathrm{Ki}-67$, the mitotic index and p53. Ki-67 is a nuclear antigen expressed in the G1, G2 and synthesis phases of the cell cycle but not in the quiescent $\mathrm{G} 0$ phase. Ki-67 is useful for predicting recurrence (3). The recent World Health Organization Tumor Classification of Tumors of Endocrine Organs defines an atypical pituitary adenoma as a tumor with a Ki-67 labeling index $>3 \%$ and extensive p53 positivity (4). No clear relationship exists between immunohistochemical p53 overexpression and recurrence or progression (5). One study reported that downregulation of the Bax protein is associated with progression of PA, whereas $\mathrm{Bcl}$ overexpression does not play a role in progression (6).

Predictive markers of recurrence and progression are yet to be firmly established. Angiogenesis is a fundamental process during the expansion of solid tumors and is also essential for progression of PA $(7,8)$. VEGF-A is one of the most important angiogenic factors stimulating endothelial cell proliferation, motility and permeability (9). VEGF-A binds to different tyrosine kinase receptors such as VEGFR-1 and VEGFR-2. These cells also express neuropilin-1 (NRP-1), a VEGFR-2 co-receptor lacking the intracellular signaling tyrosine kinase domain that enhances VEGF-A binding to VEGFR-2. VEGF selectively upregulates its homologous receptor, NRP1. VEGFinduced angiogenesis is derived not only from the direct effects on endothelial cells but also from NRP-1 upregulation stimulated by VEGF itself (10).

Here, we evaluated patients who had PAs with and without progression in a unique series that included tumor materials obtained by transsphenoidal surgery (TSS). We investigated expression of Ki-67, p53, Bax, Bcl, VEGFR-1, VEGRR-2, VEGF-A and NRP-1 in primary and recurrent 
Table I. Oligonucleotide sequences used for real-time polymerase chain reaction.

\begin{tabular}{ll} 
Gene name & \multicolumn{1}{c}{ Primer sequence $\left(5^{\prime} \rightarrow 3^{\prime}\right)$} \\
\hline NRP1 & GGAGCTACTGGGCTGTGAAG, CCTCCTGTGAGCTGGAAGTC \\
VEGF & TATCTTCAAGCCGTCCTGTG, GATCCGCATGATCTGCATAG \\
p53 & GACAGCCAAGTCTGTTATGGCAC,GACTTCTTGTAGATGGCCATGGC \\
p21 & CCTGGTGATGTCCGACCTG, CCATGAGCGCATCGCAATC \\
Bad & AAGTCCGATCCCGGAATCC, GCTCACTCGGCTC AAACTCT \\
Bax & CCCGAGCTGATCAGAACCAT, TTGGATCCAGACAAGCAGCC \\
Bid & GAGCCAGATTCTGAAAGTCAGGA, GCCTTGTCGTTCTCCATGTC \\
Bcl2 & ATGCCTTTGTGGAACTATATGGC, GGTATGCACCCAGAGTGATGC \\
Bcl211 & AGCGTAGACAAGGAGATGCAG, CCAAGGCTCTAGGTGGTCATTC \\
$18 \mathrm{~s}$ & GTAACCCGTTGAACCCCATT, CCATCCAATCGGTAGTAGC
\end{tabular}

specimens to identify a predictive marker associated with tumor progression.

\section{Materials and methods}

Patients and tumor samples. We searched the database of any patients who underwent TSS for treatment of PAs by the author (Lee S.W.) between 2002 and 2006. Cases that fulfilled the following criteria were enrolled for the study: pathology reports with a diagnosis of adenoma; medical records containing imaging reports and at least 5 years of postoperative follow-up; available pathology material. Recurrent or residual adenomas treated with radiation were excluded. Cushing's disease and growth hormone-secreting tumors were excluded.

Data from clinical notes, surgical reports and radiological findings were obtained for analyses. Morbidity, follow-up and outcome results were identified from entries in the clinical notes. Tumor location, size and relationship to neighboring anatomic structures were determined using preoperative computed tomography (CT) and magnetic resonance (MR) imaging. The invasion of cavernous sinus was determined by preoperative MR image and intraoperative inspection. The extent of tumor resection was determined intraoperatively and confirmed by follow-up MR images taken postoperatively.

Patients with deteriorating vision due to chiasmal compression by a regrowth of the tumor after an initial postoperative imaging study underwent re-TSS. Re-TSS was chosen by patients who wished to avoid the risks of radiation such as hypopituitarism. Tumor progression was defined as cases treated with re-TSS within 5 years after the first TSS and an interoperation interval $>1$ year (11). This study was approved by the institutional review board.

Immunohistochemical analysis. The protocol for $\mathrm{Ki}-67$, p53, Bcl-2, Bax, VEGF-A, VEGFR-1, VEGFR-2 and NRP-1 antibody staining has been described previously $(12,13)$. The immunostained slides were examined under light microscopy by one of the authors (Yoo C.Y.) who was blinded to the patients' clinical histories. Ki-67 was recorded as a labeling index by counting the number of immunopositive nuclei among 100 tumor cells in at least five representative high power fields across the slide. The results of p53, Bax, Bcl-2, VEGFR-1 and VEGFR-2 staining were simplified as positive (at least some stained cells) or negative. The immunohistochemical results for VEGF-A and NRP-1 were scored semi-quantitatively using a four-point scale: 0 , no immunoreaction; $1+$, faint or equivocal immunoreaction in $<10 \%$ of cells; $2+$, unequivocal, strong immunoreaction in $<30 \%$ of cells; $3+$, unequivocal, strong immunoreaction in $<60 \%$ of cells and $4+$, unequivocal, strong immunoreaction in $<100 \%$ of cells.

Statistical analysis. The distribution of patient characteristics was compared between the no progression and progression groups (treated with re-TSS), using the Wilcoxon rank-sum test and the Fisher's exact test for continuous and discrete variables, respectively. Differences in immunohistochemical results between groups were analyzed using the Chi-square test. A P-value $<0.05$ was considered significant.

Cell cultures and shRNA lentivirus production. The MMQ cell line was purchased from the American Type Culture Collection (ATCC; Manassas, VA, USA). MMQ cells were grown in F-12K, $15 \%$ horse serum, $2.5 \%$ FBS and $1 \%$ penicillin-streptomycin at $37^{\circ} \mathrm{C}$ in humidified $5 \% \mathrm{CO}_{2} / 95 \%$ air.

The human U6 promoter containing the shRNA lentiviral vector was constructed as follows: a human U6 promoter was amplified from the pLKO.1 vector (Sigma-Aldrich, St. Louis, MO, USA) by polymerase chain reaction; this fragment was placed into the CMV promoter site of the pCDH-MCS vector (\#CD513-B; System Biosciences, Palo Alto, California, CA, USA). The shRNA sequences were as follows: NRP1 shRNA sense, 5'-GCCCGAATGTTCTCAGAACTATCAAGAGTA GTTCTGAGAACATTCGGGCTTTTTT-3'; NRP1 shRNA antisense, 5'-AAAAAAGCCCGAATGTTCTCAGAACTA CTCTTGATAGTTCTGAGAACATTCGGGC-3'; control shRNA (\#TR30019, OriGene) sense, 5'-GCACTACCAGAG CTAACTCAGATAGTACTTCAAGAGAGTACTATCTGAG TTAGCTCTGGTAGTGCTTTTTT-3'; control 1 shRNA antisense, 5'-AAAAAAGCACTACCAGAGCTAACTCAG ATAGTACTCTCTTGAAGTACTATCTGAGTTAGCTCTG GTAGTGC-3'.

The shRNA lentiviruses were produced by transfecting Lenti-X 293T cells (Clontech, Palo Alto, CA, USA) with 
Table II. Clinical characteristics of the pituitary adenoma cases with and without progression within 5 years of the initial surgery.

\begin{tabular}{|c|c|c|c|}
\hline \multirow[b]{2}{*}{ Characteristics } & \multicolumn{2}{|c|}{ No. of patients (\%) } & \multirow[b]{2}{*}{ P-value } \\
\hline & $\begin{array}{l}\text { No progression } \\
\text { group }(n=19)\end{array}$ & $\begin{array}{l}\text { Progression } \\
\text { group }(n=9)\end{array}$ & \\
\hline Gender & & & 0.064 \\
\hline Male & $2(10.5)$ & $4(44.4)$ & \\
\hline Female & $17(89.5)$ & $5(55.6)$ & \\
\hline Age (years) & & & 0.369 \\
\hline Median (range) & $33(20-68)$ & $35(24-57)$ & \\
\hline \multicolumn{4}{|l|}{ Tumor size } \\
\hline$<10 \mathrm{~mm}$ & $7(36.8)$ & $0 \quad(0.0)$ & 0.035 \\
\hline $10-25 \mathrm{~mm}$ & $9(47.4)$ & $4(44.4)$ & \\
\hline$>25 \mathrm{~mm}$ & $3(15.8)$ & $5(55.6)$ & \\
\hline $\begin{array}{l}\text { Cavernous sinus } \\
\text { involvement }\end{array}$ & & & 0.035 \\
\hline+ & $4(21.1)$ & $6(66.7)$ & \\
\hline- & $15(78.9)$ & $3(33.3)$ & \\
\hline $\begin{array}{l}\text { Suprasellar } \\
\text { extension }\end{array}$ & & & 0.228 \\
\hline+ & $7(36.8)$ & $6(66.7)$ & \\
\hline- & $12(63.2)$ & $3(33.3)$ & \\
\hline Extent of resection & & & 0.409 \\
\hline Total & $13(68.4)$ & $4(44.4)$ & \\
\hline Subtotal & $6(31.6)$ & $5(55.6)$ & \\
\hline Hormonal status & & & $>0.05$ \\
\hline Non-functioning & $8(42.1)$ & $4(44.4)$ & \\
\hline Prolactinoma & $11(57.9)$ & $5(55.6)$ & \\
\hline
\end{tabular}

the shRNA lentiviral expression plasmids and the packaging plasmids (Addgene, Cambridge, MA, USA), which were pCMV-VSV-G, pMDLg/pRRE and pRSV-Rev, using Lipofectamine 2000 (Invitrogen, Carlsbad, CA, USA) reagent. Infectious shRNA lentiviruses were harvested at $48 \mathrm{~h}$ post-transfection and then used to infect MMQ cells with $8 \mu \mathrm{g} / \mathrm{ml}$ polybrene (Sigma-Aldrich). Oligonucleotide sequences used for real-time polymerase chain reaction are summarized in Table I.

\section{Results}

One hundred and forty-seven patients underwent TSS for the treatment of PAs in our institution between 2002 and 2006. We selected 28 consecutive patients who met the inclusion criteria. Nineteen patients had no progression for $>5$-years of follow-up. Nine patients had tumor progression within 5 years of their first TSS and underwent re-TSS for treatment of adenoma progression. The patient characteristics are summarized in Table II. Tumor size was larger in the progression group than that in the no progression group $(\mathrm{P}=0.035)$. Involvement of the cavernous sinus was more frequent in the progression group
Table III. Comparison of molecular marker expression between the pituitary adenoma cases with and without progression within 5 years of the initial surgery.

\begin{tabular}{|c|c|c|c|}
\hline \multirow[b]{2}{*}{ Characteristics } & \multicolumn{2}{|c|}{ No. of patients (\%) } & \multirow[b]{2}{*}{ P-value } \\
\hline & $\begin{array}{l}\text { No progression } \\
\text { group }(n=19)\end{array}$ & $\begin{array}{l}\text { Progression } \\
\text { group }(n=9)\end{array}$ & \\
\hline $\mathrm{Ki}-67$ & & & $>0.05$ \\
\hline Negative & $18(94.7)$ & $9(100)$ & \\
\hline $1-2 \%$ & $1 \quad(5.3)$ & $0(0.0)$ & \\
\hline p53 & & & 0.530 \\
\hline Negative & $16(84.2)$ & $9(100)$ & \\
\hline Positive & $3(15.8)$ & $0(0.0)$ & \\
\hline Bax & & & 0.670 \\
\hline Negative & $12(63.2)$ & $7(77.8)$ & \\
\hline Positive & $7(36.8)$ & $2(22.2)$ & \\
\hline $\mathrm{Bcl}-2$ & & & $>0.05$ \\
\hline Negative & $18(94.7)$ & $8(88.9)$ & \\
\hline Positive & $1 \quad(5.3)$ & $1(11.1)$ & \\
\hline VEGFR-1 & & & $>0.05$ \\
\hline Negative & $18(94.7)$ & $8(88.9)$ & \\
\hline Positive & $1 \quad(5.3)$ & $1(11.1)$ & \\
\hline VEGFR-2 & & & 0.530 \\
\hline Negative & $16(84.2)$ & $9(100)$ & \\
\hline Positive & $3(15.8)$ & $0(0.0)$ & \\
\hline VEGF-A & & & 0.228 \\
\hline- & $1 \quad(5.3)$ & $\begin{array}{ll}0 & (0.0)\end{array}$ & \\
\hline+ & $0(0)$ & $1(11.1)$ & \\
\hline++ & $1 \quad(5.3)$ & $2(22.2)$ & \\
\hline+++ & $7(36.8)$ & $4(44.5)$ & \\
\hline++++ & $10(52.6)$ & $2(22.2)$ & \\
\hline NRP-1 & & & 0.034 \\
\hline- & $13(68.4)$ & $5(55.6)$ & \\
\hline+ & $6(31.6)$ & $1(11.1)$ & \\
\hline++ & $0(0)$ & $0 \quad(0.0)$ & \\
\hline+++ & $0(0)$ & $3(33.3)$ & \\
\hline++++ & $0(0)$ & $0 \quad(0.0)$ & \\
\hline
\end{tabular}

Immunostaining intensity for vascular endothelial growth factor (VEGF)-A and neuropilin-1 (NRP-1) was assessed according to an arbitrary scale: -, no immunoreactivity; +, <10\%; ++, 10-30\%; +++, $31-60 \% ;++++, 61-100 \%$.

than that in the no progression group $(\mathrm{P}=0.035)$. No difference was found in regards to gender, age, suprasellar extension, or extent of resection.

Table III shows the distribution of the progression and control groups according to molecular marker expression. A strong association was observed between NRP-1 expression and tumor progression. NRP-1 immunoreactivity $3+$ and $1+$ were observed in three $(33.3 \%)$ and one patient $(11.1 \%)$, respectively, in the progression group. Thirteen patients 

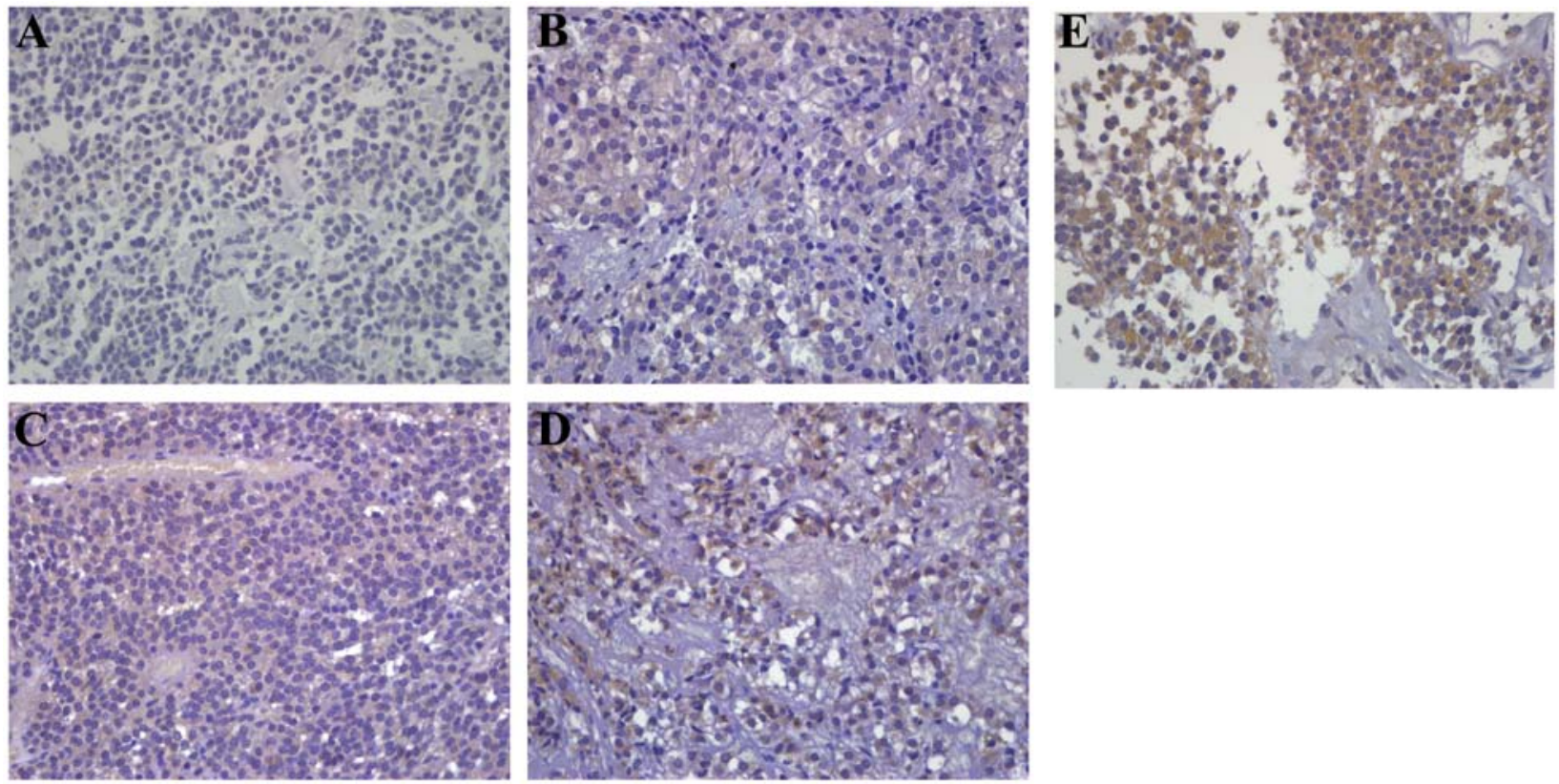

Figure 1. Neuropilin-1 (NRP-1) immunohistochemical staining (magnification, x400). Only cells with distinct cell membrane staining were considered to positively express NRP-1. The degree of NRP-1 expression was estimated semi-quantitatively as follows: (A) 0 , (B) $1+$, (C) $2+$, (D) $3+$ and (E) $4+$.
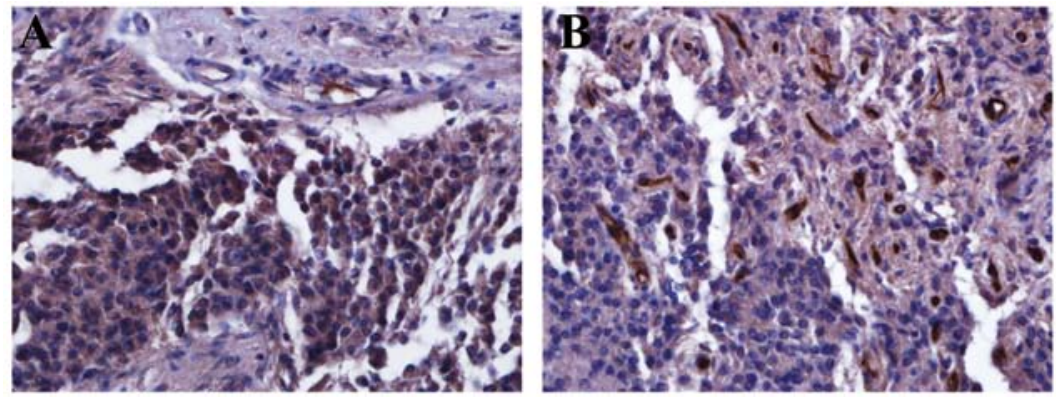

Figure 2. Neuropilin-1 (NRP-1) immunohistochemical staining in adenoma cells (A) rather than in endothelial cells showing CD31 immunoreactivity (B) (magnification, $\mathrm{x} 400$ ).

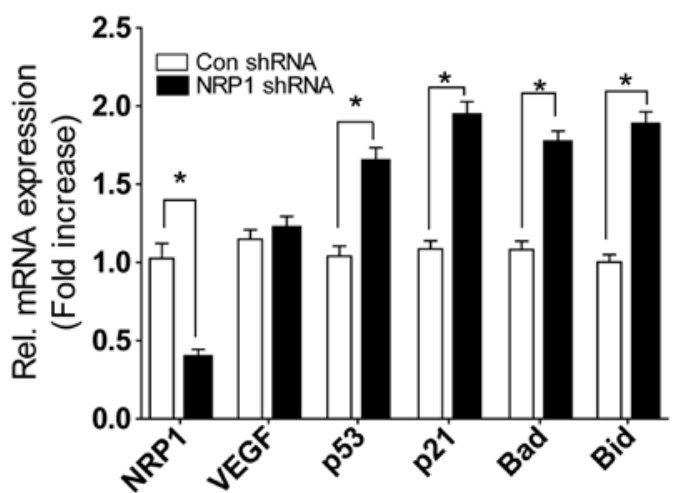

Figure 3. Knockdown of NRP-1 gene by the NRP-1 shRNA lentivirus in MMQ cells. Real-time PCR showed a significant increase in p53, p21, Bad and Bid expression and little change in VEGF expression. Data are presented as mean \pm SEM $\left(n=5\right.$ each). ${ }^{*}<<0.05$ vs. con.

(68.4\%) showed negative immunoreactivity to NRP-1 in the control group (Fig. 1). No significant risk for developing tumor progression was associated with $\mathrm{Ki}-67, \mathrm{p} 53$, Bax, Bcl-2, VEGFR-1, VEGFR-2 or VEGF-A expression.
Table IV shows a comparison of molecular marker expression in the specimens obtained during the first and second TSS in the progression group. No significant expression changes in Ki-67, p53, Bax, Bcl-2, VEGFR-1 or VEGFR-2 were observed between the initial adenomas and progressive adenomas. Five patients showed stronger VEGF-A expression in progressive adenomas, compared with that in the initial adenomas and four patients showed the same degree of immunoreactivity. Notablu, NRP-1 expression was converted from negative immunoreactivity in the initial specimens to strong positivity in the progressive specimens of five patients. One patient showed 4+ NRP-1 expression in the progressive specimen, although the initial specimen showed 1+ NRP-1 expression. Three patients showed strong NRP-1 immunoreactivity in both the initial and progressive specimens (3+). NRP-1 immunoreactivity was noted in PA cells (Fig. 2). The median interval between the first and second TSS was 28 months.

NRP-1 shRNA lentivirus significantly suppressed mRNA of NRP-1. Knockdown of NRP-1 in MMQ cells did not decrease VEGF expression, but increased the expression of p53, p21, Bad and Bid genes (Fig. 3). 
Table IV. Comparison of molecular marker expression in pituitary adenomas obtained following the first and second TSS for treatment of progression within 5 years of the initial surgery.

\begin{tabular}{|c|c|c|c|c|c|c|c|c|c|c|}
\hline $\begin{array}{l}\text { Case } \\
\text { no. }\end{array}$ & Surgery & $\mathrm{Ki}-67$ & $\mathrm{p} 53$ & Bax & $\mathrm{Bcl}$ & VEGFR-1 & VEGFR-2 & VEGF-A & NRP-1 & $\begin{array}{l}\text { Interval between the } \\
\text { first and second TSS } \\
\text { (in months) }\end{array}$ \\
\hline \multirow[t]{2}{*}{1} & First & - & - & - & - & - & - & ++ & - & 25 \\
\hline & Second & - & - & - & - & - & - & ++++ & +++ & \\
\hline \multirow[t]{2}{*}{2} & First & - & - & - & - & + & - & +++ & + & 44 \\
\hline & Second & - & - & - & - & - & - & ++++ & ++++ & \\
\hline \multirow[t]{2}{*}{3} & First & - & - & + & - & - & - & +++ & - & 18 \\
\hline & Second & - & - & + & - & - & - & ++++ & ++ & \\
\hline \multirow[t]{2}{*}{4} & First & - & - & - & - & - & - & ++++ & - & 55 \\
\hline & Second & - & - & + & + & - & - & ++++ & ++ & \\
\hline \multirow[t]{2}{*}{5} & First & - & - & + & + & - & - & ++++ & +++ & 38 \\
\hline & Second & - & + & + & + & - & - & ++++ & +++ & \\
\hline \multirow[t]{2}{*}{6} & First & - & - & - & - & - & - & +++ & - & 26 \\
\hline & Second & - & - & - & - & - & - & ++++ & ++ & \\
\hline \multirow[t]{2}{*}{7} & First & - & - & - & - & - & - & + & +++ & 30 \\
\hline & Second & - & - & - & - & - & - & ++ & +++ & \\
\hline \multirow[t]{2}{*}{8} & First & - & - & - & - & - & - & ++ & +++ & 24 \\
\hline & Second & - & - & + & - & - & - & ++ & +++ & \\
\hline \multirow[t]{2}{*}{9} & First & - & - & - & - & - & - & +++ & - & 34 \\
\hline & Second & - & + & - & - & - & - & +++ & +++ & \\
\hline
\end{tabular}

Immunostaining intensity for vascular endothelial growth factor (VEGF)-A and neuropilin-1 (NRP-1) was assessed according to an arbitrary scale: -, no immunoreactivity; +, <10\%; ++, 10-30\%; +++, 31-60\%; ++++, 61-100\%. TSS, transsphenoidal surgery.

\section{Discussion}

No acceptable markers are available to predict tumor recurrence and progression and thus aid the clinician in assessing the need for adjuvant therapy or determining further follow-up for pituitary adenomas (PAs) in the postoperative setting. In our study, a strong association was observed between NRP-1 expression and PA progression in the immunohistochemical analysis. The involvement of the cavernous sinus was associated with tumor progression. NRP-1 expression was associated with the invasion of cavernous sinus $(\mathrm{P}=0.024)$. NRP-1 could have a role in mediating the invasiveness of PAs.

NRPs are a family of transmembrane glycoprotein receptors that interact with members of the VEGF ligand family. NRP-1 is expressed in various human cancers, including prostate cancer, breast cancer, melanoma and pancreatic adenocarcinoma but not in corresponding normal epithelial tissues $(14,15)$. Those studies focused on the ability of NRP-1 to act as a co-receptor to increase the affinity of a ligand (e.g., VEGF) for a tyrosine kinase receptor (e.g., VEGF-2). Normal pituitary tissue (from normal controls) seems to display more vascularity than tissue from adenoma samples $(16,17)$. The vessels of normal tissue may be larger than those from adenomas (18). Several studies have been unable to show a relationship between VEGF expression and tumor recurrence $(19,20)$, although VEGF overexpression is associated with extrasellar growth (21). No correlation was observed between the degree of VEGF and its receptors and tumor progression parameters, such as grade, proliferation index and vessel density (22). In our series, VEGFR-1, VEGFR-2 and VEGF-A expression were not different between the progression and no progression groups in initial specimens obtained during the first TSS. Negative immunoreactivity of VEGFR-1 and VEGFR-2 was predominant in both groups. Therefore, it is suggested that the VEGF/VEGFR-1,2 system could not be directly involved in tumor progression. There was a tendency for VEGF-A overexpression in both the first and second specimens obtained during repeat TSS in the progression group. VEGF contributes to adequate tumoral vascular supply through complex mechanisms, other than tumor cell proliferation (23). Other authors have suggested that VEGF may prolong cell survival by inducing expression of the antiapoptotic protein Bcl-2 in PAs $(24,25)$. However, our results show little association between tumor progression and the expression of Bax and Bcl-2.

Increased NRP-1 expression has been correlated with tumor growth and vacularization in vivo and with invasiveness in human cancer (26). VEGF and NRP-1 actively participate in the modulation of tumor angiogenesis and the development of PAs in rats (27). In our series, NRP-1 expression was significantly associated with tumor progression. All specimens in the progression group showed negative immunoreactivity to VEGFR-2, the NRP-1 co-receptor, suggesting a VEGF-independent function for NRP-1 in NFPA progression. Experimental evidence indicates that NRP-1 may have functions independent of VEGFR-2, potentially through a NRP 
interacting protein (28). Five patients in the progression group showed negative NRP-1 immunoreactivity in their initial specimens. Interestingly, specimens obtained at the reoperation showed positive immunoreactivity. Similarly, NRP-1 is constitutively expressed in sinusoidal endothelial cells in normal liver, but its expression increases following resection of liver for sinusoidal remodeling (29). NRP-1 expression can be stimulated in response to tissue injury or hypoxic conditions $(30,31)$. We suggest that expression of NRP-1 is not static and it is affected by the tumor microenvironment.

PA transplants are effectively inhibited by administration of anti-VEGF-A monoclonal antibody (32). Anti-VEGF (bevacizumab) treatment stabilizes aggressive PAs in patients (33). However, our results showed that tumor progression was dependent on NRP-1 expression rather than that of VEGF. Antitumor activity of monoclonal antibody to NRP-1 is currently evaluated in cancers (34). We generated an shRNA lentivirus against NRP-1 to determine the effects of in vitro NRP-1 knockdown. Knockdown of NRP-1 increased the expression of apoptotic genes such as p53, p21, Bad and Bid. This indicates that downregulation of NRP-1 can induce the apoptosis of PA cells, independently of VEGF expression. Taken together, we suggest that NRP-1 is considered as a novel therapeutic target for PAs.

\section{Acknowledgements}

The authors wish to acknowledge the financial support of the St. Vincent's Hospital, Research Institute of Medical Science Foundation in 2014.

\section{References}

1. Ezzat S, Asa SL, Couldwell WT, et al: The prevalence of pituitary adenomas: a systematic review. Cancer 101: 613-619, 2004.

2. Hsu DW, Hakim F, Biller BM, et al: Significance of proliferating cell nuclear antigen index in predicting pituitary adenoma recurrence. J Neurosurg 78: 753-761, 1993.

3. Gejman R, Swearingen B and Hedley-Whyte ET: Role of Ki-67 proliferation index and p53 expression in predicting progression of pituitary adenomas. Hum Pathol 39: 758-766, 2008.

4. Lloyd RV: Pituitary tumors. In: World Health Organization Classification of Tumors, Pathology and Genetics of Tumors of Endocrine Organs. DeLellis A (ed.). IARC Press, Lyon, pp10-13, 2004.

5. Hentschel SJ, McCutcheon 1E, Moore W and Durity FA: P53 and MIB-1 immunohistochemistry as predictors of the clinical behavior of nonfunctioning pituitary adenomas. Can J Neurol Sci 30: 215-219, 2003.

6. Sambaziotis D, Kapranos N and Kontogeorgos G: Correlation of bcl-2 and bax with apoptosis in human pituitary adenomas. Pituitary 6: 127-133, 2003.

7. Iuchi T, Saeki N, Osato K and Yamaura A: Proliferation, vascular endothelial growth factor expression and cavernous sinus invasion in growth hormone secreting pituitary adenomas. Acta Neurochir 142: 1345-1351, 2000.

8. Cohen $\mathrm{AB}$ and Lessell S: Angiogenesis and pituitary tumors Semin Ophthalmol 24: 185-189, 2009.

9. Tammela T, Enholm B, Alitalo K and Paavonen K: The biology of vascular endothelial growth factors. Cardiovasc Res 65: 550-563, 2005

10. Oh H, Takagi H, Otani A, et al: Selective induction of neuropilin-1 by vascular endothelial growth factor (VEGF): a mechanism contributing to VEGF-induced angiogenesis. Proc Natl Acad Sci USA 99: 383-388, 2002.

11. Benveniste RJ, King WA, Walsh J, et al: Repeated transsphenoidal surgery to treat recurrent or residual pituitary adenoma. J Neurosurg 102: 1004-1012, 2005.
12. Lee JS, Yoon A, Kalapurakal SK, et al: Expression of p53 oncoprotein in non-small-cell lung cancer: a favorable prognostic factor. J Clin Oncol 13: 1893-1903, 1995.

13. Thunnissen FB, Schuurbiers OC and den Bakker MA: A critical appraisal of prognostic and predictive factors for common lung cancers. Histopathology 48: 779-786, 2006.

14. Bielenberg DR, Pettaway CA, Takashima S and Klagsbrun M: Neuropilins in neoplasms: expression, regulation, and function. Exp Cell Res 312: 584-593, 2006.

15. Guttmann-Raviv N, Kessler O, Shraga-Heled N, et al: The neuropilins and their role in tumorigenesis and tumor progression. Cancer Lett 231: 1-11, 2006.

16. Turner HE, Nagy Z, Gatter KC, et al: Angiogenesis in pituitary adenomas and the normal pituitary gland. J Clin Endocrinol Metab 85: 1159-1162, 2000.

17. Di Ieva A, Grizzi F, Ceva-Grimaldi G, et al: Fractal dimension as a quantitator of the microvasculature of normal and adenomatous pituitary tissue. J Anat 211: 673-680, 2007.

18. Takada K, Yamada S and Teramoto A: Correlation between tumor vascularity and clinical findings in patients with pituitary adenomas. Endocr Pathol 15: 131-139, 2004.

19. Fukui S, Nawashiro H, Otani N, et al: Vascular endothelial growth factor expression in pituitary adenomas. Acta Neurochir Suppl 86: 519-521, 2003.

20. Fukui S, Otani N, Nawashiro $\mathrm{H}$, et al: The association of the expression of vascular endothelial growth factor with the cystic component and haemorrhage in pituitary adenoma. J Clin Neurosci 10: 320-324, 2003.

21. Sánchez-Ortiga R, Sánchez-Tejada L, Moreno-Perez O, et al: Over-expression of vascular endothelial growth factor in pituitary adenomas is associated with extrasellar growth and recurrence. Pituitary 16: 370-377, 2013.

22. Onofri C, Theodoropoulou M, Losa M, et al: Localization of vascular endothelial growth factor (VEGF) receptors in normal and adenomatous pituitaries: detection of a non-endothelial function of VEGF in pituitary tumours. J Endocrinol 191: 249-261, 2006.

23. Cristina C, Perez-Millan MI, Luque G, et al: VEGF and CD31 association in pituitary adenomas. Endocr Pathol 21: 154-160, 2010.

24. Nör JE, Christensen J, Mooney DJ and Polverini PJ: Vascular endothelial growth factor (VEGF)-mediated angiogenesis is associated with enhanced endothelial cell survival and induction of Bcl-2 expression. Am J Pathol 154: 375-384, 1999.

25. Turner HE, Nagy Z, Gatter KC, Esiri MM, et al: Proliferation, bcl-2 expression and angiogenesis in pituitary adenomas: relationship to tumour behaviour. Br J Cancer 82: 1441-1445, 2000.

26. Pan Q, Chanthery Y, Liang WC, et al: Blocking neuropilin-1 function has an additive effect with anti-VEGF to inhibit tumor growth. Cancer Cell 11: 53-67, 2007.

27. Banerjee SK, Zoubine MN, Tran TM, et al: Overexpression of vascular endothelial growth factor164 and its co-receptor neuropilin-1 in estrogen-induced rat pituitary tumors and GH3 rat pituitary tumor cells. Int J Oncol 16: 253-260, 2000.

28. Pan Q, Chathery Y, Wu Y, et al: Neuropilin-1 binds to VEGF121 and regulates endothelial cell migration and sprouting. J Biol Chem 282: 24049-24056, 2007.

29. Fu L, Kitamura T, Iwabuchi K, Ichinose S, et al: Interplay of neuropilin-1 and semaphorin $3 \mathrm{~A}$ after partial hepatectomy in rats. World J Gastroenterol 18: 5034-5041, 2012.

30. Matthies AM, Low QE, Lingen MW and DiPietro LA: Neuropilin-1 participates in wound angiogenesis. Am J Pathol 160: 289-296, 2002.

31. Brusselmans K, Bono F, Collen D, et al: A novel role for vascular endothelial growth factor as an autocrine survival factor for embryonic stem cells during hypoxia. J Biol Chem 280: 3493-3499, 2005.

32. Korsisaari N, Ross J, Wu X, et al: Blocking vascular endothelial growth factor-A inhibits the growth of pituitary adenomas and lowers serum prolactin level in a mouse model of multiple endocrine neoplasia type 1. Clin Cancer Res 14: 249-258, 2008.

33. Ortiz LD, Syro LV, Scheithauer BW, et al: Anti-VEGF therapy in pituitary carcinoma. Pituitary 15: 445-449, 2012.

34. Jubb AM, Strickland LA, Liu SD, et al: Neuropilin-1 expression in cancer and development. J Pathol 226: 50-60, 2012. 